\title{
Feasibility of the modified sequential organ function assessment score in a resource-constrained setting: a prospective observational study
}

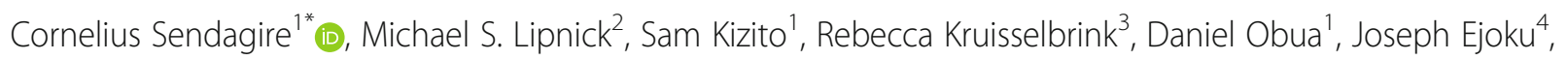
Lameck Ssemogerere ${ }^{1}$, Jane Nakibuuka ${ }^{4}$ and Arthur Kwizera ${ }^{1}$

\begin{abstract}
Background: Sub-Saharan Africa has a great burden of critical illness with limited health care resources. We evaluated the feasibility and utility of the modified Sequential Organ Function Assessment (mSOFA) score in assessing morbidity and mortality in the National Referral Hospital's intensive care unit (ICU) for one year.

Methods: We conducted a prospective, observational cohort study on patients above 12 years of age admitted to the ICU at Mulago Hospital (Kampala, Uganda). All SOFA scores were determined at admission and at $48 \mathrm{~h}$. We modified the SOFA score by replacing the $\mathrm{PaO}_{2} / \mathrm{FiO}_{2}$ ratio with $\mathrm{SPO}_{2} / \mathrm{FiO}_{2}$. The primary outcome was ICU mortality.

Results: This ICU cohort of 118 patients had a mean age of 37 years and an ICU mortality rate of $46.6 \%$. Non-survivors had higher initial (7.7 SD 3.8 vs. 5.5 SD 3.3; $p=0.007$ ), mean (8.1 SD 3.9 vs 4.7 SD 2.6; $p<0.001$ ) and highest mSOFA scores (9.4 SD 4.2 vs. 5.8 SD 3.2; $p<0.001$ ), with an increase of 1.0 (SD 3.1) mSOFA on average after $48 \mathrm{~h}$ when compared to survivors $(p<0.001)$. The area under the receiver operating characteristic curves for each mSOFA category was: initial-0.68, mean-0.76, highest- 0.76 and delta mSOFA-0.74. Multivariate logistic regression analysis showed no significant association between mSOFA scores and mortality.
\end{abstract}

Conclusion: Our results confirm that calculation of the mSOFA score is feasible for an ICU population in a resource-limited country. More data are needed to test for an association between mSOFA and mortality.

Keywords: Modified sequential organ function assessment, Mortality, Low and middle-income countries, Illness severity scoring system, Critical care

\section{Background}

Critical illness in low and middle income countries (LMICs) is a major component of the global burden of disease [1]. These countries have relatively younger patient populations than those of high income countries (HICs) [2,3]. Despite this, ICU patients in LMICs still have more morbidity and mortality than those in HICs largely due to limited resources and the severity of co-morbid conditions [1-4].

\footnotetext{
* Correspondence: cornels77@gmail.com

${ }^{1}$ Department of Anesthesia and critical care „Makerere University College of Health Sciences, P.O. Box 7072, Kampala, Uganda

Full list of author information is available at the end of the article
}

In 1994, the European Society of Intensive Care Medicine (ESICM) committee developed the Sequential Organ Failure Assessment (SOFA) score 5, 6]. This score assesses the functionality of six organ systems (Table 1) [7]. It was validated as a tool for assessment of organ dysfunction and mortality prediction in ICU [8]. While simpler than many other tools used to predict ICU mortality, the application of SOFA still requires several lab tests (platelets, bilirubin, creatinine and blood gas analysis) $[6,8,9]$. The availability of such lab tests in LMICs is limited thereby limiting the widespread applicability of this score [1]. Some studies have validated modifications of the SOFA score in which certain lab tests were replaced with more readily 
Table 1 Sequential Organ Function Assessment score

\begin{tabular}{|c|c|c|c|c|c|}
\hline SOFA score & 0 & 1 & 2 & 3 & 4 \\
\hline \multicolumn{6}{|l|}{ Respiratory } \\
\hline $\mathrm{PaO}_{2} / \mathrm{FiO}_{2}$ & $>400$ & $300-400$ & $200-300$ & $100-200$ & $<100$ \\
\hline $\mathrm{SPO}_{2} / \mathrm{FIO}_{2}^{\mathrm{a}}$ & $>301$ & $221-301$ & $142-220$ & $67-141$ & $<67$ \\
\hline \multicolumn{6}{|l|}{ Coagulation } \\
\hline Platelets $10^{3} / \mathrm{mm}^{3}$ & $>150$ & $<150$ & $<100$ & $<50$ & $<20$ \\
\hline \multicolumn{6}{|l|}{ Liver } \\
\hline Bilirubin (mg/dL) & $<1.2$ & $1.2-1.9$ & $2.0-5.9$ & $6.0-11.9$ & $>12.0$ \\
\hline \multicolumn{6}{|l|}{ Cardiovascular } \\
\hline \multirow[t]{2}{*}{ Hypotension } & No & MAP & Dopamine & Dopamine & Dopamine \\
\hline & hypotension & $<70$ & $\begin{array}{l}</=5 \mathrm{ug} / \mathrm{kg} / \mathrm{min} \text { or } \\
\text { dobutamine (any dose) }\end{array}$ & $\begin{array}{l}>5 \mathrm{ug} / \mathrm{kg} / \mathrm{min} \text { or norepinephrine } \\
</=0.1 \mathrm{ug} / \mathrm{kg} / \mathrm{min}\end{array}$ & $\begin{array}{l}>15 \mathrm{ug} / \mathrm{kg} / \mathrm{min} \text { or } \\
\text { norepinephrine }>0.1 \mathrm{ug} / \mathrm{kg} / \mathrm{min}\end{array}$ \\
\hline \multicolumn{6}{|l|}{ CNS } \\
\hline Glasgow Coma & 15 & $13-14$ & $10-12$ & $6-9$ & $<6$ \\
\hline \multicolumn{6}{|l|}{ Score } \\
\hline \multicolumn{6}{|l|}{ Renal } \\
\hline $\begin{array}{l}\text { Creatinine }(\mathrm{mg} / \mathrm{dL}) / \text { urine } \\
\text { output }(\mathrm{ml} / \mathrm{d})\end{array}$ & $<1.2$ & $1.2-1.9$ & $2.0-3.4$ & $3.5-4.9$ or $<500$ & $>5.0$ or $<200$ \\
\hline
\end{tabular}

$\mathrm{SPO}_{2}$ : Peripheral saturation of oxygen; $\mathrm{FiO}_{2}$ : Fraction of inspired Oxygen; MAP: Mean Arterial Pressure; CNS; Central Nervous System

${ }^{a}$ Modification to include $\mathrm{SPO}_{2} / \mathrm{FIO}_{2}$ as a replacement for $\mathrm{PaO}_{2} / \mathrm{FiO}_{2}$

available clinical measurements [10-12]. The SOFA score, for example, requires blood gas analysis for calculating the $\mathrm{PaO}_{2} / \mathrm{FiO}_{2}$ ratio [5]. However, a study in a $\mathrm{HIC}$ validated the use of $\mathrm{SpO}_{2} / \mathrm{FiO}_{2}$ ratio as a surrogate for the $\mathrm{PaO}_{2} / \mathrm{FiO}_{2}$ [10]. This modification was hypothesized to increase feasibility in settings where blood gas measurements are not regularly available. However, this modified SOFA score has not been validated in LMICs. In another study in a HIC, a modified SOFA score requiring only one laboratory measurement was validated to assess feasibility for critical care triage during pandemics [11]. In that study, Grissom et al. eliminated platelet count, substituted $\mathrm{SpO} 2$ for $\mathrm{PaO} 2$, replaced bilirubin with icterus and did bedside point-of-care creatinine testing. The only study to date from a low-income country was conducted in an urban hospital in Nepal and validated the use of the SOFA score but without any modification [12].

Due to low specificity of the SIRS criteria, the most recent sepsis guidelines have incorporated SOFA score into the new sepsis definition $[13,14]$. Unlike SIRS criteria however, the SOFA requires more laboratory evaluations which limit its feasibility in LMICs [9].

With a paucity of literature aimed at addressing critical care in LMICs, we conducted a study to determine the feasibility and utility of using a modified SOFA (mSOFA) Score for assessing organ dysfunction and predicting mortality in a tertiary hospital ICU of a lowincome country.

\section{Methods}

\section{Study area and setting}

We conducted a prospective, observational cohort study for one year (February, 2014 - January, 2015) in the ICU of Mulago Hospital (Kampala, Uganda). Mulago Hospital is a 1500-bed teaching hospital and the national referral hospital for Uganda. The hospital's inpatient census often exceeds 2000 and has a large proportion of critically-ill patients [15]/ The hospital's ICU has eight beds although only four beds are equipped with ventilators. The ICU is staffed by two intensivists, one senior resident, one to two anesthesia residents per day, and nurses. It has a nurse to patient ratio of 1:2 - 1:4. Complete blood counts, as well as liver and renal function tests are routinely available; however, blood gas analysis is rarely available. The ICU admits between 200-300 critically-ill patients annually with an ICU mortality of approximately $40 \%$ [16].

\section{Study population}

Study approval was granted by the Makerere University School of Medicine Research and Ethics Committee. A written informed consent/assent was obtained from next of kin prior to enrollment or a waiver of consent was obtained for those without next of kin. All patients $>12$ years of age admitted to the Mulago Hospital ICU for more than $24 \mathrm{~h}$ were enrolled. We chose a lower limit of 13 years because the SOFA score has not been validated among patients $<13$ years [5]. The exclusion criteria were admission for low risk monitoring, death 
or discharge in the first $24 \mathrm{~h}$ and no outcome at the end of the study.

\section{Study procedure}

We collected demographic data, all vital signs, complete blood count, as well as liver and renal function tests for each patient at admission and at $48 \mathrm{~h}$ after admission. Blood gas analysis was to be done with the ABL 80 Flex model in the ICU. The mSOFA score, ranging from 0 (normal organ function) to 24 (worst organ dysfunction), was calculated on admission $\left(\mathrm{T}_{0}\right)$ and at $48 \mathrm{~h}\left(\mathrm{~T}_{48}\right)$. Patients were then followed until ICU discharge or the end of the study period. In calculating the mSOFA score, the worst values for each parameter in the 24-h period were used. The $\mathrm{SPO}_{2} / \mathrm{FiO}_{2}$ ratio was used instead of $\mathrm{PaO}_{2} / \mathrm{FiO}_{2}$ ratio, and the remaining parameters were used based on the calibration as shown in Table 1 . We selected the range cutoffs shown in Table 1 as these ranges had been validated in two prior studies. For patients on nasal cannula oxygen, we estimated $\mathrm{FiO}_{2}$ by multiplying the liter flow per minute by 0.03 and adding that to 0.21 . For a single missing data point, a replacement was estimated by calculating the mean of the sum of the results immediately preceding and following the missing value. The total mSOFA score was calculated as the sum of the mSOFA score at admission and at 48-h. The initial mSOFA score was the average $\mathrm{T}_{0}$ of all patients. The mean mSOFA score was defined as the average of mSOFA score over the two days of calculation of the mSOFA while in the ICU. The highest recorded SOFA score was also recorded as the highest between the two recording times. The delta SOFA score was defined as the difference between $\mathrm{T}_{0}$ and $\mathrm{T}_{48}$. After discharge or death, data were crosschecked for completeness and accuracy with patient's chart prior to data entry by a trained staff member.

\section{Statistical analysis}

The primary outcome was ICU mortality or discharge. We compared continuous variables of the survivors and non-survivors using the student $t$-test with a significant $p$-value of $<0.05$. We used the chi-square test for categorical variables with a significant $p$-value of $<0.05$. Odd ratios with $95 \%$ confidence interval were computed using a multivariate logistic regression model with ICU mortality as the dependent variable. The predictive ability of the SOFA scores was evaluated using receiver operating characteristic (ROC) curve analysis generated on STATA 12 (Statacorp College Station, TX). The area under the receiver operating characteristic curve (AUROC) was used to compare the discriminatory power of the scoring system, with an AUC 1.0 considered perfect discrimination and 0.5 considered equal to chance. Numerical continuous data were summarized as means, standard deviations for the normally distributed data, and as medians and interquartile ranges for the non-normally distributed data. Categorical data were summarized with percentages and proportions.

\section{Results}

From February 20, 2014 to January 31, 2015 we screened 201 patients, and enrolled 170. Subsequently, 51 patients were excluded. Of these, 42 of them were admitted for only 24-h of postoperative monitoring, nine died within $24 \mathrm{~h}$ of admission and only one had no outcome at the end of the study (Fig. 1). There were nine non-adults

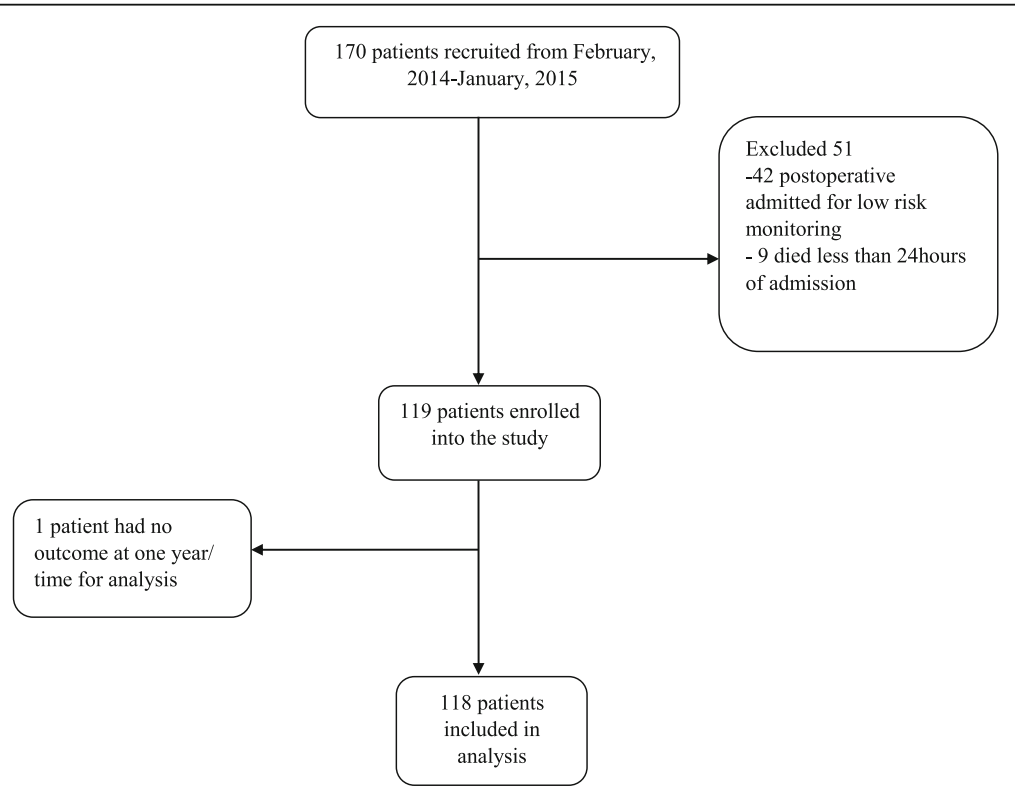

Fig. 1 Enrolment 
(ages 13-18) in the cohort, all of whom had informed consent obtained from their appropriate family members. We thus analyzed 118 patients (58\% male) with a mean (SD) age of 37 (17) years and an ICU mortality rate of $46.6 \%$. During the study period, $\mathrm{PaO}_{2}$ values were missing in $90 \%$ of the study patients while there was no missing value for bilirubin, creatinine and platelet count. The median length of stay in the ICU was six days (IQR; 3-10) while the median survival time within the ICU was 12 days (95\% CI 6.38-17.62). Sepsis prevalence according to the SIRS criteria was $50 \%$ on admission to the ICU. Table 2 summarizes the demographics and initial clinical characteristics of the study cohort.

The initial, mean, highest and delta mSOFA scores (SD) were: 6.5 (3.7), 6.3 (3.7), 7.5 (4.1) and 0.4 (3.2) respectively. Non-survivors had significantly higher initial, mean and higher mSOFA scores than survivors: 7.7 vs. $5.5(p=0.001), 8.1$ vs. $4.7(p<0.001)$ and 9.4 vs. 5.8 $(p<0.001)$ respectively. We also found that while the initial mSOFA score decreased by 1.7 in survivors; that of non-survivors increased by 1.0 after $48 \mathrm{~h}(p<0.001)$. On admission, only the respiratory and cardiovascular
mSOFA scores of non-survivors were significantly higher than those of survivors (Table 3). The same trend was observed at $48 \mathrm{~h}$, however, the central nervous system and renal mSOFA scores were also significantly higher in non-survivors.

The adjusted odds ratios of the mSOFA scores for mortality were not statistically significant despite trends showing direct proportionality with mortality as seen in Fig. 2 and neither was there a statistical difference in their slopes found. The 48-h change in mSOFA score also had no significant association with mortality $p$-value $=0.61$.

Using AUROC for discriminating mortality, the initial mSOFA score had the lowest predictive power with an AUC of 0.68 (95\% CI, 0.58-0.77) while the mSOFA at $48 \mathrm{~h}$ had the highest predictive power of 0.79 (95\% CI, 0.71-0.87). The mean, highest and delta mSOFA scores demonstrated a fairly accurate prediction with AUROC: 0.76 (95\% CI, 0.68-0.85), 0.76 (95\% CI, 0.66-0.85), and 0.74 (95\% CI, 0.65-0.84) respectively (Fig. 3 and Table 4). We also found that the mean mSOFA score AUC was significantly higher than that of the initial mSOFA with a $p$ value of 0.0003 .

Table 2 Demographics and clinical characteristics of 118 critically patients admitted to mulago hospital icu from February 2014 to January 2015

\begin{tabular}{|c|c|c|c|c|}
\hline Characteristics & All & Survivors & Non-Survivors & $P^{a}$ \\
\hline Age (years); mean (SD) & $37.7(17.5)$ & $37.7(17.4)$ & $37.7(17.7)$ & 0.99 \\
\hline \multicolumn{5}{|l|}{ Sex } \\
\hline Male (\%) & $68(57.6)$ & $41(66.7)$ & $25(47.3)$ & 0.033 \\
\hline \multicolumn{5}{|l|}{ Source (\%) } \\
\hline Emergency room & $33(28)$ & $19(30.2)$ & $14(25.4)$ & \\
\hline Operating room & $21(17.8)$ & $15(23.8)$ & $6(10.9)$ & \\
\hline PACU & $2(1.7)$ & $2(3.2)$ & $0(0.0)$ & \\
\hline Obstetric theatre & $3(2.5)$ & $0(0.00)$ & $3(5.5)$ & \\
\hline Medical ward & $25(21.2)$ & $9(15.9)$ & $15(27.3)$ & \\
\hline Surgical ward & $11(9.3)$ & $6(9.5)$ & $5(9.1)$ & \\
\hline Obstetric-gynecology ward & $7(5.9)$ & $2(3.2)$ & $5(9.1)$ & \\
\hline Other hospital & 16 (13.6) & $9(14.3)$ & $8(12.7)$ & 0.117 \\
\hline Medical Admissions (\%) & $40(33.9)$ & $20(31.8)$ & $20(36.4)$ & 0.597 \\
\hline \multicolumn{5}{|l|}{ HIV status (\%) } \\
\hline Positive & $12(10.1)$ & $6(9.5)$ & $6(10.8)$ & \\
\hline Negative & $88(74.6)$ & $48(77.8)$ & 41 (73.6) & \\
\hline Unknown & $18(15.3)$ & $8(12.7)$ & $9(16.2)$ & 0.664 \\
\hline Mechanical Ventilation (\%) & $100(84.8)$ & $47(47.0)$ & $53(53.0)$ & 0.001 \\
\hline Ventilation time (SD) (hours) & $159.07(247.87)$ & $156.05(227.95)$ & $162.53(270.99)$ & 0.888 \\
\hline Vasopressor use (\%) & $28(23.7)$ & $4(14.2)$ & $24(85.7)$ & 0.008 \\
\hline Length of stay (Days);mean (SD) & $10.2(12.8)$ & $12.5(13.8)$ & $7.5(11.1)$ & 0.033 \\
\hline No. of patients (\%) & 118 & $63(53.4)$ & $55(46.6)$ & \\
\hline
\end{tabular}

PACU: Post anaesthetic care unit; SD: Standard deviation; HIV: Human immunodeficiency virus; ICU: Intensive care unit; $A B G$ : Arterial blood gas $P^{a}$ values calculated using unpaired $t$ tests, Mann-Whitney $U$ tests, or chi-square tests where appropriate 
Table 3 Calculated sofa scores and comparisons of 118 critically ill-patients admitted to mulago hospital icu from February 2014 to January 2015

\begin{tabular}{lllll}
\hline SOFA score & All & Survivors & Non-survivors & $P^{a}$ \\
\hline SOFA-0 mean (SD) & & & & \\
SOFA-RS & $1.6(1.2)$ & $1.3(1.2)$ & $1.9(1.2)$ & 0.007 \\
SOFA-CVS & $0.6(1.2)$ & $0.2(0.7)$ & $1(1.6)$ & 0.001 \\
SOFA-CNS & $2.8(1.1)$ & $2.8(1.2)$ & $2.8(1.1)$ & 0.66 \\
SOFA-Liver & $0.3(0.8)$ & $0.2(0.7)$ & $0.4(0.8)$ & 0.168 \\
SOFA-Coagulation & $0.3(0.7)$ & $0.3(0.6)$ & $0.4(0.7)$ & 0.364 \\
SOFA-renal & $1(1.5)$ & $0.8(1.4)$ & $1.2(1.6)$ & 0.115 \\
Total SOFA & $6.5(3.7)$ & $5.5(3.3)$ & $7.7(3.8)$ & 0.001 \\
SOFA-48 mean (SD) & & & & \\
SOFA-RS & $1.2(1.2)$ & $0.7(0.9)$ & $1.8(1.4)$ & $<0.001$ \\
SOFA-CVS & $0.7(1.4)$ & $0.1(0.4)$ & $1.4(1.8)$ & $<0.001$ \\
SOFA-CNS & $2.6(1.4)$ & $2(1.3)$ & $3.4(1.0)$ & $<0.001$ \\
SOFA-Liver & $0.2(0.6)$ & $0.2(0.6)$ & $0.3(0.6)$ & 0.379 \\
SOFA-Coagulation & $0.3(0.7)$ & $0.3(0.6)$ & $0.4(0.7)$ & 0.620 \\
SOFA-renal & $1(1.5)$ & $0.7(1.4)$ & $1.4(1.6)$ & 0.007 \\
Total SOFA & $6.0(4.3)$ & $3.9(2.6)$ & $8.5(4.6)$ & $<0.001$ \\
Mean SOFA (SD) & $6.3(3.7)$ & $4.7(2.6)$ & $8.1(3.9)$ & $<0.001$ \\
Highest SOFA (SD) & $7.5(4.1)$ & $5.8(3.2)$ & $9.4(4.2)$ & $<0.001$ \\
Delta SOFA (SD) & $0.4(3.2)$ & $-1.7(2.7)$ & $1.0(3.1)$ & $<0.001$ \\
\hline SOFA: Sequential organ & $f u n c t o n$ & assessment; RS: & \\
Sespiratory system; &
\end{tabular}

SOFA: Sequential organ function assessment; RS: Respiratory system; CVS: Cardiovascular system; CNS: Central nervous system

$P^{a}$ values calculated using unpaired $t$ tests, Mann-Whitney $U$ tests

\section{Discussion}

The present study is the first in Sub-Saharan Africa to determine the feasibility and utility of modifying the SOFA score for evaluating organ dysfunction and critical care mortality in a resource-limited ICU. In our study we were able to calculate mSOFA for all patients even though we were unable to prove that it is a good predictor of mortality. Since $90 \%$ were missing $\mathrm{PaO}_{2}$ values; majority of patients in our study did not have data required to calculate a traditional SOFA score.

The use of simple, inexpensive and rapid methods to assess illness severity and predict outcomes is important in all clinical settings, especially those in low-income countries where resources are limited and illness severity is high $[1,4,6,11]$. Recently the specificity of SIRS criteria for sepsis has come into question and led to the development of new sepsis definitions that now include the SOFA score $[13,14,17,18]$. Although the Third Consensus Definitions for Sepsis and Septic Shock includes a discussion of quick SOFA (qSOFA) scores, the vast majority of efforts to simplify or validate scoring tools (like SOFA) take place almost exclusively in highincome countries.

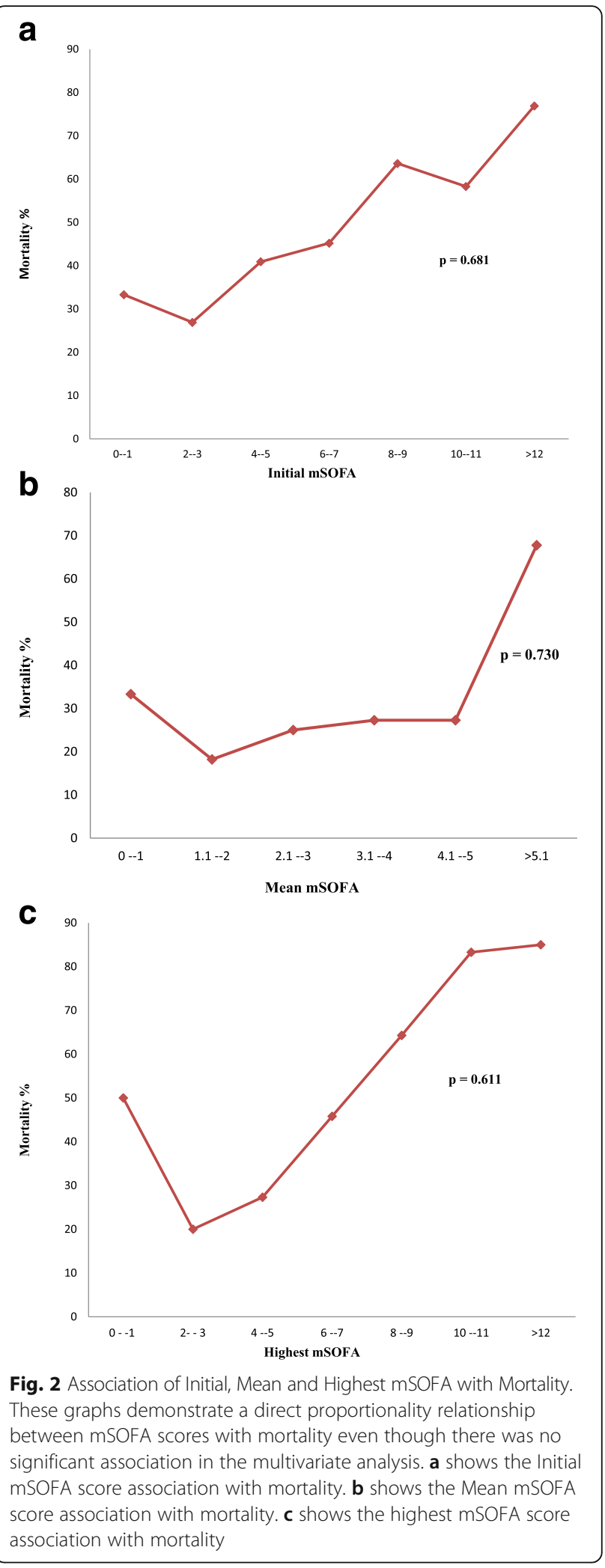

The initial mSOFA score for our study was less than that of a study from HIC and the only other study from a LIC; Nepal (6.5 vs. 7.1 vs. 7.9 respectively) $[7,12]$. The 


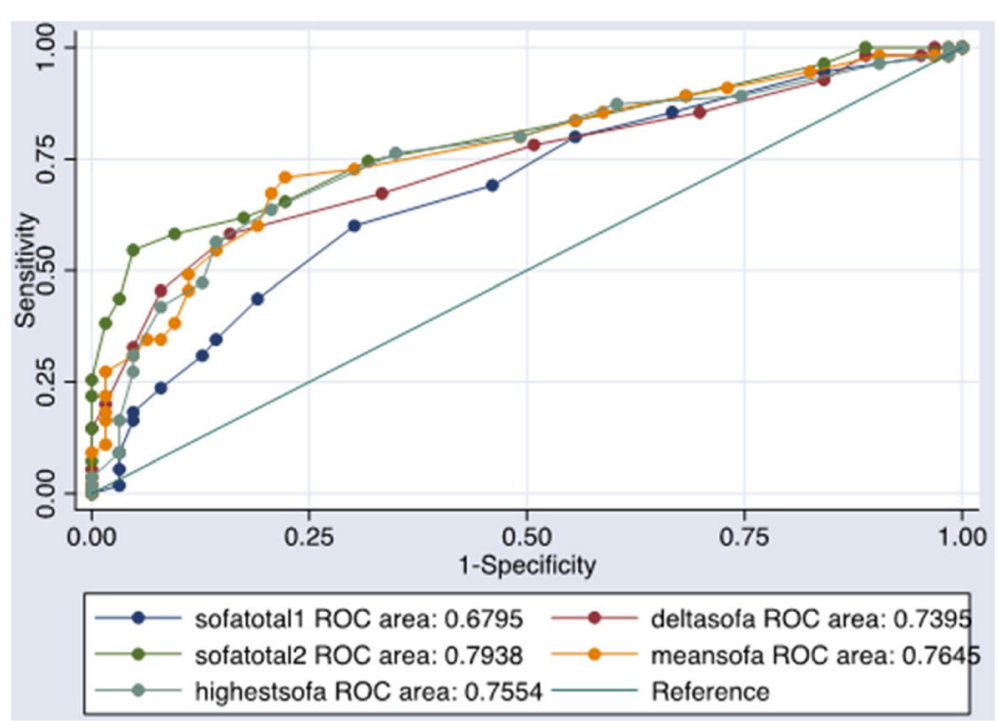

Fig. 3 Receiver Operating Curves for mSOFA prediction of mortality. The mSOFA demonstrated a poor to fair prediction of mortality. The initial mSOFA score is represented by Sofatotal1. It showed the lowest predictive power of all categories of the mSOFA. The mSOFA at $48 \mathrm{~h}$, represented by Sofatotal2, had the highest predictive power followed by the mean, highest and delta mSOFA scores

Nepalese study is the only prior study of SOFA scores in a LIC and utilized the original SOFA score in an urban hospital (six bed ICU) [12]. That study found significant predictive value of SOFA scores for mortality using univariate analysis. Similar to our study, Acharya et al. also had a relatively young patient cohort (mean age 34 years) with a high mortality rate (40\%). Significant differences between our studies exist and include the relatively smaller sample size, better hospital resources (e.g. regular availability of blood gas analyses) and inclusion only of patients who met the SIRS criteria for the Nepalese study. Our initial mSOFA was lower than that from Acharya despite our population having higher mortality rate overall. This suggests that our $\mathrm{SpO} 2$ modification may underestimate the respiratory variable of the mSOFA score as discussed in greater detail below.

The results of prior studies of modified SOFA scores in HICs may not be applicable in LICs due to marked differences in clinical environments and patient populations $[4,11]$. In the current study we used the $\mathrm{SPO}_{2} / \mathrm{FiO}_{2}$ ratio as a surrogate for the $\mathrm{PaO}_{2} / \mathrm{FiO}_{2}$ in calculating the

Table 4 Mortality predictive power of the msofa score for 118 critically ill-patients admitted to mulago hospital icu from February 2014 to January 2015

\begin{tabular}{lll}
\hline mSOFA & AUROC & $95 \%$ Confidence interval \\
\hline Initial & 0.68 & $0.58-0.78$ \\
$48 \mathrm{~h}$ & 0.79 & $0.71-.88$ \\
Mean & 0.76 & $0.68-0.85$ \\
Highest & 0.76 & $0.67-0.85$ \\
Delta & 0.74 & $0.65-0.84$ \\
\hline
\end{tabular}

SOFA score, a modification previously validated but only in HICs $[10,11]$. Overall, the $\mathrm{PaO}_{2}$ values were missing in a very large percentage of our patient despite the fact that $48 \%$ had at least one blood gas analysis done during the ICU stay. This $48 \%$ was still a relatively larger proportion of patients with blood gas done than hypothesized, but due in large part to a concurrent observational study that provided cartridges for the blood gas machine stationed in the ICU. We found that the $\mathrm{PaO}_{2}$ values were missing even in those who had blood gas analysis done because it was done outside the recording times: $\mathrm{T}_{0}$ and $\mathrm{T}_{48}$.

Compared to ICUs of HICs, those in LICs have a mortality rate nearly four times higher largely due to inadequate resources $[1,2,4,12,16,19]$. The mortality rate in our study was relatively higher than that found in a prior study done in the same ICU by Kwizera et al. and Acharya's study in Nepal: 47 vs. 43 vs. $40 \%$ respectively $[12,16]$. Kwizera et al's study had no exclusion criteria unlike our study, which could have led to their slightly lower mortality rate. The Nepalese study was conducted in an ICU that was better resourced than our own and was conducted on patients who met the SIRS criteria. Since positive SIRS criteria has been associated with a higher mortality rate [13], it is highly likely that this was the reason their mortality was nearly as high as that found in our study.

We found that all patients in the present study had mSOFA scores more than twice the designated cut off for organ dysfunction as outlined by the The Third International Consensus Definitions for Sepsis and Septic Shock [14]. Similar to other studies, the mSOFA scores of non-survivors in our cohort were significantly 
higher than those in survivors $[5,7,8,12,19,20]$. We also found that the initial mSOFA score had the lowest AUROC compared to the mean, highest and delta mSOFA scores, similar to prior investigations $[8,12,21]$. With exception of the initial mSOFA, the other mSOFAs demonstrated fairly accurate prediction however the confidence intervals were wide and thus ought to be interpreted with caution. This was also supported by the finding that the mean mSOFA had a significantly higher predictability than the initial mSOFA. Our univariate analysis did show predictive value for the initial mSOFA yet this relationship did not hold true with multivariate analysis. Of note, the prior study in Nepal undertook only univariate analysis that showed significant associations [12]. Even though we found no significant association between the MSOFA and mortality, Fig. 2 shows a linear relationship if a line of best fit was drawn for each of the mSOFA categories. We still however, did not find any statistical significance of any of the slopes in any combination. We believe this was due to the small sample size such that occurrence of mortality in any of the ranges we used for each mSOFA category resulted into a bigger percentage of that category than would have been otherwise.

Prior studies have found that SOFA score is most predictive in the first $48 \mathrm{~h}$ and can be used to assess degree of organ dysfunction, mortality and response to therapy throughout admission [8]. Although our results were unable to corroborate all of these findings, this study provides evidence that further validation and modification of the most recent sepsis definitions is feasible in LICs and warranted. The utility of a simple validated tool for assessing and predicting outcomes in LICs would have far-reaching applicability that extends beyond patient care and also includes future research to improve outcomes in these settings.

There were several limitations with the present study. The first and most significant was low power due to small sample size. Due to limited access to medical records and limited study personnel, we were unable to enroll and analyze data for patients admitted for less than $24 \mathrm{~h}$. This resulted in the exclusion of a large number of potential study subjects. Another significant limitation of the current study may be attributable to the modification of SOFA scores using $\mathrm{SpO}_{2}$ in our setting. There are several reasons the $\mathrm{SpO}_{2} / \mathrm{FiO}_{2}$ calculation may not accurately reflect severity of pulmonary dysfunction. For example, in order to be categorized in the sickest mSOFA cohort by $\mathrm{SpO}_{2}$, a patient would need to have a $\mathrm{SpO} 2$ of $<67 \%$ while on $100 \%$ $\mathrm{FiO}_{2}$. Only three patients in our cohort met this criterion. Our use of $\mathrm{SpO}_{2}$ likely underestimated severity of pulmonary dysfunction. For example, a patient with an $\mathrm{SpO}_{2}$ of $90 \%$ on $100 \% \mathrm{FiO} 2$ would be given 3 points using the mSOFA (90/1 =90; Table 3). Using the original SOFA score and assuming that same patient has a $\mathrm{PaO}_{2}$ of $\sim 60$, the patient would be given 4 points $(60 / 1=60$; Table 3$)$. An additional significant limitation of $\mathrm{SpO}_{2} / \mathrm{FiO}_{2}$ calculations in our setting is the lack of reliable oxygen sources. For example, tank and wall oxygen frequently are less than $100 \%$ FiO2 (anecdotally as low as 70\%). This could result in significant overestimation of severity of pulmonary dysfunction. In future investigations, the investigation of multiple $\mathrm{SpO}_{2}$ respiratory thresholds may yield improved predictive value for the mSOFA Score. An additional limitation that we must recognize due to our single site design is the possibility for site bias. We performed the mSOFA score on only two occasions, $48 \mathrm{~h}$ apart, limiting the information on organ dysfunction assessment over the length of stay with treatment. The generalizability to austere environments outside our study setting is challenging, as further investigation and more robust study resources are needed.

\section{Conclusion}

Our results confirm that calculation of the mSOFA score is feasible for an ICU population in a low-income country. Although the present study did not demonstrate a definitive relation between mSOFA and mortality, larger studies are needed to assess the discriminative power of mSOFA for predicting organ dysfunction and mortality in resource-limited settings. Such data may be able to help better characterize critical illness disease burden and the triage of limited resources.

\section{List of abbreviations \\ APACHE: Acute Physiologic And Chronic Health Evaluation; CNS: Central Nervous System; ESICM: European Society of Intensive Care Medicine; $\mathrm{FiO}_{2}$ : Fraction of inspired Oxygen; GSK: GlaxoSmithKline; HICs: High Income Countries; ICU: Intensive Care Unit; IQR: Interquartile Range; LMICs: Lower Middle Income Countries; mSOFA: modified Sequential Organ Function Assessment Score; $\mathrm{PaO}_{2}$ : Arterial Partial pressure of Oxygen; qSOFA: quick Sequential Organ Function Assessment; SAPS: Simple Acute Physiologic Score; SD: Standard Deviation; SIRS: Systemic Inflammatory Response \\ Syndrome; SOFA: Sequential Organ Function Assessment; $\mathrm{SPO}_{2}$ : Peripheral Saturation of Oxygen; $T_{0}$ : mSOFA at admission (initial SOFA Score); \\ $\mathrm{T}_{48}$ : mSOFA at $48 \mathrm{~h}$}

\section{Acknowledgements}

The authors appreciate the support from, Mulago Hospital, the Department of Anaesthesia-Makerere University, Global Partnership in Anesthesia and Surgery, Association of Anaesthetists of Great Britain and Ireland and the Ministry of Health of Uganda.

\section{Funding}

None.

\section{Availability of data and materials}

The datasets generated during and/or analysed during the current study are not publicly available due to the on-going further analysis on other variables with mortality collected during the study but are available from the corresponding author on reasonable request.

\section{Authors' contributions}

CS, LS conceived the study. CS, MSL, DO, JE, JN and AK developed the protocol. CS collected the data. SK and RK did the data management and statistics. Interpretation was done by CS, MSL, AK, JN and SK. CS, MSL and AK drafted the manuscript. All authors helped with manuscript drafting and approved the final manuscript. 


\section{Competing interests}

The authors declare that they have no competing interests.

\section{Consent for publication}

Not applicable.

\section{Ethics approval and consent to participate}

Informed consent was obtained from each patient. If the patient was not able to provide consent on admission, it was obtained from the next of kin If there was no next of kin at the time of admission to the ICU, a waiver of consent from the IRB had been granted to allow patient data collection.

\section{Author details}

'Department of Anesthesia and critical care ${ }^{\prime}$ Makerere University College of Health Sciences, P.O. Box 7072, Kampala, Uganda. ${ }^{2}$ San Francisco General Hospital, University of California San Francisco, 1001 Potrero, 3C24, San Francisco, CA 94110, USA. ${ }^{3}$ McMaster University, 1280 Main St W, Hamilton, ON L8S 4L8, Canada. ${ }^{4}$ Mulago National Referral Hospital, P.O. Box 7051, Kampala, Uganda.

Received: 4 September 2016 Accepted: 17 January 2017

Published online: 26 January 2017

\section{References}

1. Dunser M, Baelani WI, Ganbold L. A review and analysis of intensive care medicine in the least developed countries. Crit Care Med. 2006:34:1234-42.

2. Adhikari NK, Fowler RA, Bhagwanjee S, Rubenfeld GD. Critical care and the global burden of critical illness in adults. Lancet. 2010;376:1339-46.

3. Fowler RA, Adhikari NKJ, Bhagwanjee S. Clinical review: critical care in the global context - disparities in burden of illness, access, and economics. Crit Care. 2008:12(225):1-6.

4. Murthy S, Wunsch $\mathrm{H}$. Clinical review: International comparisons in critical care - lessons learned. Crit Care. 2012;16(218):1-7.

5. Vincent J, de Mendonça A, Cantraine F, Moreno R, Takala J, Sprung CL. Use of the SOFA score to assess the incidence of organ dysfunction/failure in intensive care units: results of a multicenter, prospective study. Working group on "sepsis-related problems" of the european society of intensive care medicine. Crit Care Med. 1998;26(11):1793-800.

6. Vincent JL, Moreno R, Takala J, Willatts S, De Mendonca A, Bruining H, Reinhart CK, Suter PM, Thijs LG. The SOFA (Sepsis.related Organ Failure Assessment) score to describe organ dysfunction/failure On behalf of the Working Group on Sepsis Related Problems of the European Society of Intensive Care Medicine. Intensiv Care Med. 1996;22:707-710

7. Jones AE, Trzeciak S, Kline JA. The sequential organ failure assessment score for predicting outcome in patients with severe sepsis and evidence of hypoperfusion at the time of emergency department presentation. Crit Care Med. 2009;37(5):1649-54.

8. Ferreira LF, Bota DP, Bross A, Melot C, Vincent JL. Serial evaluation of the SOFA score to predict outcome in critically III patients. JAMA. 2001;286(14):1754-58.

9. Bouch DC, Thompson JP. Severity scoring systems in the critically ill. Contin Educ Anaesth Crit Care Pain. 2008;8(5):181-5.

10. Pandharipande PP, Shintani AK, Hagerman HE, St Jacques PJ, Rice TW, Sanders NW, Ware LB. Derivation and validation of Spo2/Fio2 ratio to impute for Pao2/Fio2 ratio in the respiratory component of the sequential organ failure assessment score. Crit Care Med. 2009:37(4):1317-21.

11. Grissom CK, Brown SM, Kuttler KG, Boltax JP, Jones J, Jephson AR, Orme Jr JF. A modified sequential organ failure assessment (MSOFA) score for critical care triage. Disaster Med Public Health Prep. 2010;4(4):1-17.

12. Acharya SP, Pradhan B, Marhatta MN. Application of "the sequential organ failure assessment (SOFA) score" in predicting outcome in ICU patients with SIRS. Kathmandu Univ Med J. 2007:5(20):475-83.

13. Kaukonen K, Bailey M, Pilcher D, Cooper DJ, Bellomo R. Systemic Inflammatory Response Syndrome Criteria in Defining Severe Sepsis. NEJM. 2015;372:1-10.

14. Singer M, Deutschman CS, Seymour CW, Shankar-Hari M, Annane D, Bauer M, Bellomo R, Bernard GR, Chiche JD, Coopersmith CM, Hotchkiss RS, Levy MM, Marshall JC, Martin GS, Opal SM, Rubenfield GD, Poll TV, Vincent JL, Angus DC. The third international consensus definitions for sepsis and septic shock (sepsis-3). JAMA. 2016;315(8):801-10.

15. Kruisselbrink R, Kwizera A, Crowther M, Fox AR, O'Shea T, Nakibuuka J, Ssinabulya I, Nalyazi J, Bonner A, Devji T, Wong J, Cook D. Modified early warning score (MEWS) identifies critical illness among ward patients in a resource restricted setting in Kampala, Uganda: a prospective observational study. PLoS One. 2016;10:1-13.

16. Kwizera A, Dunser M, Nakibuuka J. National intensive care unit bed capacity and ICU patient characteristics in a low income country. BMC Res Notes. 2012;5(475):1-6

17. Seymour CW, Liu VX, Iwashyna TJ, Brunkhorst FM, Rea TD, Scherag A, Rubenfield G, Kahn JM, Shankar-Hari M, Singer M, Deutschman CS, Escobar GJ, Angus DC. Assessment of clinical criteria for sepsis for the third international consensus definitions for sepsis and septic shock (sepsis-3). JAMA. 2016:315(8):762-74.

18. Shankar-Hari M, Phillips GS, Levy ML, Seymour CW, Liu VX, Deutschman CS, Angus DC, Rubenfield GD, Singer M. Developing a NewDefinition and assessing New clinical criteria for septic shock for the third international consensus definitions for sepsis and septic shock (sepsis-3). JAMA. 2016; 315(8):775-87.

19. Vosylius S, Sipylaite J, Ivaskevicius J. Sequential organ failure assessment score as the determinant of outcome for patients with severe sepsis. Croat Med J. 2004;45(6):715-20.

20. Moreno R, Vincent JL, Matos R, Mendonça A, Cantraine F. The use of maximum SOFA score to quantify organ dysfunction/failure in intensive care. Results of a prospective, multicentre study. Working group on sepsis related problems of the ESICM. Intensive Care Med. 1999;25(7):686-96.

21. Minne L, Abu-Hanna A, de Jonge E. Evaluation of SOFA-based models for predicting mortality in the ICU: a systematic review. Crit Care. 2008;12(6):1-13.

\section{Submit your next manuscript to BioMed Central and we will help you at every step:}

- We accept pre-submission inquiries

- Our selector tool helps you to find the most relevant journal

- We provide round the clock customer support

- Convenient online submission

- Thorough peer review

- Inclusion in PubMed and all major indexing services

- Maximum visibility for your research

Submit your manuscript at www.biomedcentral.com/submit
Biomed Central 